\title{
Analytical solutions for distributed multipolar vortex equilibria on a sphere
}

\author{
Darren Crowdy a) and Martin Cloke ${ }^{\mathrm{b})}$ \\ Department of Mathematics, Imperial College of Science, Technology and Medicine, 180 Queen's Gate, \\ London SW7 2BZ, United Kingdom
}

(Received 22 April 2002; accepted 24 September 2002; published 21 November 2002)

\begin{abstract}
Analytical solutions of the steady Euler equations corresponding to stationary multipolar vortices on a sphere are derived. The solutions represent localized regions of distributed vorticity consisting of uniform vortex patches with a finite set of superposed point vortices. The mathematical method combines stereographic projection with conformal mapping theory to generalize a class of exact solutions for planar multipolar vortices developed by Crowdy [Phys. Fluids 11, 2556 (1999)] to the physically more important scenario of multipolar vortices on a spherical surface. The solutions are believed to be the first examples of analytical solutions of the Euler equations on a sphere involving patches of distributed vorticity with nontrivial shape. (c) 2003 American Institute of Physics.
\end{abstract}

[DOI: $10.1063 / 1.1521727$ ]

\section{INTRODUCTION}

In modeling large-scale features of atmospheres and oceans, it is natural to study the dynamics of vorticity on the surface of a sphere. When modeling planetary-scale coherent structures the curvature of the planet can play an important part in the dynamics. The atmosphere contains localized large-scale structures the dynamics of which can be significantly affected by the curvature of the sphere and rotational effects (as, for example, in the dynamics of concentrated regions of vorticity such as a hurricane on time scales of the order of one day). In terms of localized vorticity distributions on a sphere, point vortex models represent the most-studied paradigm (see, for example, Kidambi and Newton ${ }^{1}$ which includes an extensive list of references). The purpose of this paper is to construct and study a class of stationary coherent vortical equilibria on the surface of the sphere. The full curvature effects of the sphere are taken into account and the solutions involve localized regions of distributed uniform vorticity. The theory is at present limited to a sphere that is nonrotating.

In comparison with planar vortex equilibria, the understanding of vortical equilibria on a spherical surface is much more limited. The equations of motion for the simplest point vortex models appear to have been first written down by Bogomolov. ${ }^{2}$ Kimura and Okamoto $^{3}$ later retrieved these equations and also those relevant for the motion of patches of uniform vorticity. Dritschel and Polvani ${ }^{4}$ have used numerical contour surgery codes to study the roll-up of vorticity strips on a spherical surface and later studied multivortex equilibria modeled as patches of uniform vorticity. ${ }^{5}$ DiBattista and Polvani ${ }^{6}$ have constructed models combining point vortices and uniform vortex patches to study barotropic vortex pairs on a rotating sphere. More general models of baro-

a)Telephone: (020) 7594 8587; fax: (020) 7594 8517. Electronic mail: d.crowdy@ic.ac.uk

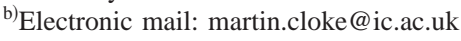

tropic vortex pairs include the exact modon solutions of Verkley. ${ }^{7}$

Recently, much attention has been paid to a class of coherent vortical structures known as multipolar vortices. A tripole, for example, has a central region with vorticity of one sign and two satellite vortices of opposite sign and such a structure has been observed in the Bay of Biscay. ${ }^{8}$ A quadrupolar vortex has a central core surrounded by three equal satellites of opposite signature; higher-order multipolar structures have correspondingly greater numbers of satellite vortices. Much recent effort has been made to understand these coherent structures (see, e.g., Van Heijst and Kloosterziel, ${ }^{9}$ Carnevale and Kloosterzial, ${ }^{10}$ Morel and Carton ${ }^{11}$ ) but most theoretical studies have been confined to two-dimensional models in the plane. In an attempt to understand these structures as a class of mathematical solutions to the twodimensional Euler equations, Crowdy ${ }^{12}$ has constructed exact solutions of these equations having all the qualitative features of the general class of multipolar equilibria. This analysis rests on a generalization of a basic solution referred to in Ref. 12 as the shielded Rankine vortex. This simple solution is a point vortex of one sign situated at the center of a uniform circular vortex patch of opposite sign such that the total circulation of the structure is zero. It might equivalently be thought of as a shielded point vortex. The generalized solutions consist of uniform vortex patches of nontrivial shape with distributions of superposed point vortices. Many of these equilibria have recently been shown to be linearly stable structures. ${ }^{13}$ The general ideas underlying this study have been extended in various directions. ${ }^{14,15}$

This paper examines whether the exact multipolar solutions of Crowdy ${ }^{12}$ can be generalized to the physically more important case of vortices on the surface of a sphere. There are two fundamental theoretical differences in finding vortical equilibria in the plane and on the sphere. The first difference is that the surface of a sphere has a nonzero curvature. The radius of curvature of the sphere defines a characteristic lengthscale not present in the planar case. The second differ- 
ence is that the sphere is a closed compact surface. As a consequence of Gauss' theorem, the integral of the scalar vorticity field over the spherical surface must, therefore, be zero. This constraint does not exist in the planar case.

Given these differences, it is by no means immediate that the exact solution structure of the planar multipolar vortex solutions of Crowdy ${ }^{12}$ will be generalizable to the sphere. Indeed, it is a well-known fact that the classical planar elliptical vortex solution of Kirchhoff ${ }^{16}$ cannot be generalized, in exact mathematical form, to a spherical surface. Strictly speaking, even the simple mathematical idealization of an isolated point vortex solution (i.e., a $\delta$-function distribution of vorticity) does not generalize directly to a sphere. This is a consequence of the constraint that the total integral of the vorticity of the sphere is zero. Instead, a $\delta$-function point vortex distribution of vorticity must always be embedded in a background of constant uniform vorticity of exactly the right strength to render the global integral of vorticity equal to zero. Such a situation is strongly reminiscent of the shielded Rankine vortex solution that has formed the basis of a general constructive method for vortical equilibria of Crowdy. ${ }^{12,14,15}$ This similarity provides a first clue that generalization of Ref. 12 to a sphere might be possible. This paper shows that a generalization can indeed be made and gives details of the construction.

We summarize the main result. In Crowdy ${ }^{12}$ it was demonstrated that streamfunctions of the form

$\psi(z, \bar{z})=\left\{\begin{array}{l}-\frac{\omega_{0}}{4}\left[z \bar{z}-\int{ }^{z} S\left(z^{\prime}\right) d z^{\prime}-\int^{\bar{z}} \bar{S}\left(z^{\prime}\right) d z^{\prime}\right] \quad z \in \bar{D}, \\ 0 \quad z \notin D\end{array}\right.$,

where $z=x+i y$ and $\bar{z}=x-i y$ denote points in a complex plane, comprise a class of exact solutions for multipolar vortices provided that $\partial D$, the boundary of the vortex patch $D$, is chosen appropriately and $S(z)$ is chosen to be the Schwarz function associated with $\partial D$. In this paper, this class of solutions is generalized to multipolar vortices on the surface of a sphere. This is done by first projecting the vortex patch stereographically onto a region $D_{p}$ of a complex $\zeta$-plane. The boundary of the vortex patch projects down to a curve $\partial D_{p}$. It is shown herein that the generalization of (1) is

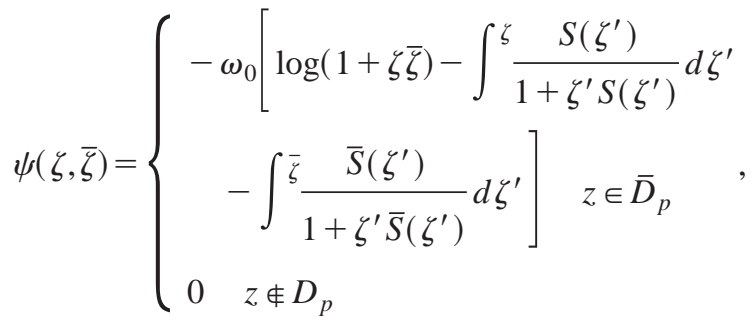

where $\bar{\zeta}$ is the complex conjugate variable to $\zeta$. For special choices of the bounding curve $\partial D_{p}$, the streamfunction (2) describes, in closed form, a class of multipolar vortices in equilibrium on the surface of a sphere.

\section{VORTEX MOTION ON A SPHERE}

Consider vortex motion on a sphere. Without loss of generality it is assumed that the sphere has unit radius. In terms of standard spherical polar coordinates $(r, \theta, \phi)$ with the latitude angle $\theta$ measured from the axis through the north pole, the velocity vector has the form

$$
\underline{u}=(0, v, u),
$$

where $u$ and $v$ are the zonal and meridional components of the velocity field, respectively. The incompressible nature of the flow allows the introduction of a scalar streamfunction $\psi$ via

$$
\underline{u}=\nabla \psi \wedge \underline{e}_{r},
$$

where $\underline{e}_{r}$ is the radial unit vector. It is then possible to define a scalar vorticity field $\omega(\theta, \phi)$ such that

$$
\omega \underline{e}_{r}=\nabla \wedge \underline{u},
$$

where

$$
\omega=-\nabla_{\Sigma}^{2} \psi,
$$

and $\nabla_{\Sigma}^{2}$ denotes the spherical Laplace-Beltrami operator

$$
\nabla_{\Sigma}^{2} \equiv \frac{1}{\sin \theta} \frac{\partial}{\partial \theta}\left(\sin \theta \frac{\partial}{\partial \theta}\right)+\frac{1}{\sin ^{2} \theta} \frac{\partial^{2}}{\partial \phi^{2}} .
$$

In terms of the streamfunction $\psi, u$, and $v$ are given as

$$
\begin{aligned}
& u=-\frac{\partial \psi}{\partial \theta}, \\
& v=\frac{1}{\sin \theta} \frac{\partial \psi}{\partial \phi} .
\end{aligned}
$$

There exists a global constraint on the vorticity distribution. Gauss' theorem dictates that only vorticity fields which integrate to zero over the sphere are permitted, i.e.,

$$
\int_{\text {sphere }} \omega d \sigma=0 \text {, }
$$

where $d \sigma$ denotes the area element on a spherical surface.

\section{STEREOGRAPHIC PROJECTION}

The analysis of Crowdy ${ }^{12}$ depends crucially on considering complexified equations of motion. The surface of a sphere can be endowed with a complex structure obtained by stereographic projection. In what follows, we consider a class of multipolar vortices centred on the south pole. A stereographic projection in which the north pole maps to infinity in a projected $\zeta$-plane is therefore appropriate. Figure 1 shows a schematic illustrating this projection onto a complex $\zeta$-plane through the equator. In polar form

$$
\zeta=r e^{i \phi},
$$

where

$$
r=\cot \left(\frac{\theta}{2}\right) .
$$

The origin $\zeta=0$ corresponds to the south pole of the sphere. It is convenient to observe that

$$
\cos \theta=\frac{\zeta \bar{\zeta}-1}{\zeta \bar{\zeta}+1},
$$




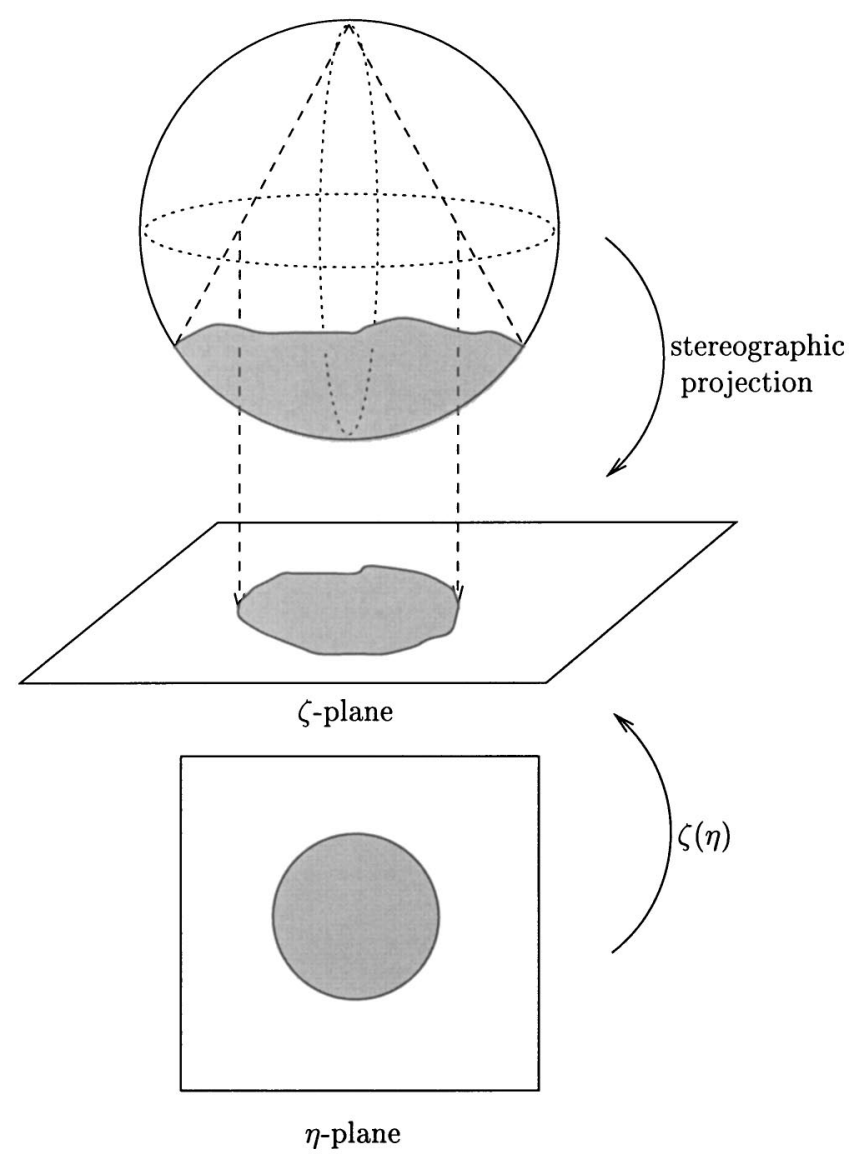

FIG. 1. Schematic illustrating stereographic projection from the physical sphere to a complex $\zeta$-plane and conformal mapping to the $\zeta$-plane from the unit-disc in a complex $\eta$-plane.

$$
\sin \theta=\frac{2 \sqrt{\zeta \bar{\zeta}}}{\zeta \bar{\zeta}+1}
$$

It can be verified that

$$
\left.\frac{\partial}{\partial \theta}\right|_{\phi}=-\left.\frac{\zeta}{\sin \theta} \frac{\partial}{\partial \zeta}\right|_{\bar{\zeta}}-\left.\frac{\bar{\zeta}}{\sin \theta} \frac{\partial}{\partial \bar{\zeta}}\right|_{\zeta}
$$

and

$$
\left.\frac{\partial}{\partial \phi}\right|_{\theta}=\left.i \zeta \frac{\partial}{\partial \zeta}\right|_{\bar{\zeta}}-\left.i \bar{\zeta} \frac{\partial}{\partial \bar{\zeta}}\right|_{\zeta} .
$$

Using (13) and (14), simple algebraic manipulations reveal that

$$
\nabla_{\Sigma}^{2} \psi=(1+\zeta \bar{\zeta})^{2} \psi_{\zeta \bar{\zeta}}
$$

where subscripts denote partial differentiation. Using (13) and (14) it follows that

$$
u-i v=\frac{2 \zeta}{\sin \theta} \psi_{\zeta}
$$

\section{MOTIVATION}

In Crowdy, ${ }^{12}$ a solution of the Euler equation which was dubbed a shielded Rankine vortex was introduced. This so- lution corresponds to the classical Rankine vortex (a circular vortex patch in pure solid body rotation ${ }^{16}$ ) with the additional feature that a single point vortex, of equal but opposite circulation to the vortex patch, is placed at the center of the Rankine vortex thus producing a composite monopolar vortex structure with zero total circulation. In terms of the standard complex coordinate $z=x+i y$, the streamfunction associated with the shielded Rankine vortex can be written

$$
\psi(z, \bar{z})=\left\{\begin{array}{l}
-\frac{\omega}{4}(z \bar{z}-2 \log |z|) \quad|z| \leqslant 1 \\
0 \quad|z|>1
\end{array},\right.
$$

where $\omega$ is the uniform patch vorticity and the Rankine vortex is assumed to have unit radius. For purposes of generalization, in Ref. 12 the observation was made that $\psi$, as given in (17), can be rewritten as

$$
\psi(z, \bar{z})=\left\{\begin{array}{l}
-\frac{\omega}{4}\left(z \bar{z}-\int^{z} S\left(z^{\prime}\right) d z^{\prime}-\int^{\bar{z}} \bar{S}\left(z^{\prime}\right) d z^{\prime}\right) \quad|z| \leqslant 1 \\
0 \quad|z|>1
\end{array},\right.
$$

where

$$
S(z)=\frac{1}{z}
$$

is the Schwarz function ${ }^{17}$ of the boundary circle $|z|=1$ of the Rankine vortex. Instead of calling the solution (17) a "shielded Rankine vortex," it is equally sensible to refer to it as a "shielded point vortex," i.e., a point vortex at the origin is being shielded by a uniform background of constant vorticity such that the total circulation of the combined structure is zero.

The latter perspective is instructive when comparing with the notion of a point vortex on a sphere. This is because, owing to the constraint (9) that the global integral of the vorticity over the sphere must always equal zero, in order to generalize the notion of a point vortex solution to the sphere, any single point vortex existing on the sphere must necessarily be shielded. The accepted way to perform this shielding is to place the singular point vortex in a background of uniform vorticity covering the whole spherical surface. ${ }^{2,3}$ Such a configuration is more reminiscent of the shielded point vortex solution (17) rather than the regular planar point vortex solution.

To see the analogy mathematically, in Appendix A the streamfunction associated with a single point vortex on a sphere is derived using the equations presented in Sec. II. In terms of the complex stereographic coordinate $\zeta$ introduced in Sec. III, the streamfunction for a point vortex of strength $-\omega / 2$ placed at the south pole is given by

$$
\psi=-\frac{\omega}{4}(\log (1+\zeta \bar{\zeta})-2 \log |\zeta|) .
$$

Equation (20) should be compared with (17). Identifying the two complex coordinates $z$ and $\zeta$, the streamfunctions take a very similar form. In (17), the term $z \bar{z}$ corresponds to the 
uniform vorticity contribution while $\log (1+\zeta \bar{\zeta})$ performs this role in (20). The slightly different functional forms are a result of the nonzero curvature effects of the sphere. Meanwhile, the point vortex contributions $\log |z|$ and $\log |\zeta|$ clearly take exactly the same functional form on both the plane and the sphere. The close analogy between (17) and (20) leads us to suggest that (17) is the natural candidate for the singular planar point vortex solution which generalizes to a singular spherical point vortex solution.

While Crowdy ${ }^{12}$ gives the generalization of the (monopolar) solution (17) to planar multipolar vortices of higher order, this paper gives the analogous generalization of the spherical monopolar solution (20).

\section{EXACT MULTIPOLAR VORTICES ON A SPHERE}

Consider a simply connected patch of uniform vorticity on the surface of the sphere. Let $D$ denote this vortex patch and let $D_{p}$ denote the stereographic projection of all points in the domain $D$ onto the $\zeta$-plane. Assume that the boundary $\partial D$ of the vortex patch projects onto a curve $\partial D_{p}$ in the $\zeta$-plane and that $\partial D_{p}$ is an analytic curve. The closure of the (open) domain $D_{p}$ in the $\zeta$-plane is denoted $\bar{D}_{p}$. Define a streamfunction having the form

$$
\psi(\zeta, \bar{\zeta})=\left\{\begin{array}{c}
-\omega_{0}\left(\log (1+\zeta \bar{\zeta})-\int^{\zeta} \mathcal{S}\left(\zeta^{\prime}\right) d \zeta^{\prime}\right. \\
\left.-\int^{\bar{\zeta}} \overline{\mathcal{S}}\left(\zeta^{\prime}\right) d \zeta^{\prime}\right) \quad \zeta \in \bar{D}_{p} \\
0 \quad \zeta \notin \bar{D}_{p}
\end{array},\right.
$$

where $\mathcal{S}(\zeta)$ is to be specified shortly. First observe that substitution of (21) into (15) shows that it satisfies the equation

$$
\nabla_{\Sigma}^{2} \psi=-\omega_{0},
$$

except possibly at any singularities of $\mathcal{S}(\zeta)$ inside the vortex patch.

The fact that $\partial D_{p}$ is an analytic curve implies that there exists, in an annular neighborhood of the curve $\partial D_{p}$, an analytic function $S(\zeta)$ such that

$$
\bar{\zeta}=S(\zeta), \quad \text { on } \partial D_{p} .
$$

The function $S(\zeta)$ is known as the Schwarz function of $\partial D_{p} \cdot{ }^{17}$ Now pick $\mathcal{S}(\zeta)$ as follows:

$$
\mathcal{S}(\zeta)=\frac{S(\zeta)}{(1+\zeta S(\zeta))},
$$

then, the form of the streamfunction becomes

$$
\psi(\zeta, \bar{\zeta})=\left\{\begin{array}{rl}
-\omega_{0}\left[\log (1+\zeta \bar{\zeta})-\int \frac{S\left(\zeta^{\prime}\right)}{1+\zeta^{\prime} S\left(\zeta^{\prime}\right)} d \zeta^{\prime}\right. \\
\left.-\int \frac{\bar{\zeta}\left(\zeta^{\prime}\right)}{1+\zeta^{\prime} \bar{S}\left(\zeta^{\prime}\right)} d \zeta^{\prime}\right] \quad z \in \bar{D}_{p} \\
0 \quad z \notin \bar{D}_{p}
\end{array} .\right.
$$

Consider the velocity field given by (16). For a consistent equilibrium solution, it is necessary that the fluid velocity is continuous at the patch boundary $\partial D$. This implies that it must be continuous on $\partial D_{p}$. It is immediate, by differentiation of (25) with respect to $\zeta$ and use of (16), that the streamfunction (25) satisfies this condition. It is required, in addition, that the boundary of the vortex patch is a streamline, i.e., a $\psi$-contour. But on $\partial D_{p}$, it can be verified that (25) satisfies the equation

$$
d \psi=\psi_{\zeta} d \zeta+\psi_{\bar{\zeta}} d \bar{\zeta}=0,
$$

which implies that $\partial D_{p}$ is indeed a streamline. The choice of streamfunction (25), therefore, satisfies both the kinematic and dynamic conditions at the vortex jump on the patch boundary.

There is the additional constraint that the global circulation on the sphere is zero. Because the region around the north pole of the sphere is stagnant, it is clear that the global circulation on the sphere is equal to the circulation around the contour $\partial D_{p}$. But the velocity field vanishes everywhere on $\partial D_{p}$ so it is easy to see that this circulation is zero. Hence the global circulation vanishes, as required.

However, for an arbitrary patch $D$, the Schwarz function of the projected curve $\partial D_{p}$ will be singular in $D_{p}$ and hence so will the composite function $\mathcal{S}(\zeta)$. This will mean that the streamfunction (25) will possess (possibly unphysical) singularities inside the vortex patch. However, to model multipolar vortices (and to obtain physically consistent solutions), consider the possibility of restricting to a special class of vortex patch domains $D$ with the property that the Schwarz function of the projected curve $\partial D_{p}$ is such that the composite function $\mathcal{S}(\zeta)$ as defined by (24) has only simple pole singularities with real residues. For this special class of domains, the streamfunction (25) will possess a finite distribution of point vortex singularities. In addition to this constraint on the vortex patch domains there exists the additional requirement, dictated by the Helmholtz laws of vortex motion, that any and all such point vortices are steady under the effects of the nonself-induced velocity field. This condition is necessary for a consistent equilibrium solution of the dynamical equations and imposes an additional constraint on the admissible class of vortex patches.

\section{A. Conformal mapping}

It turns out that such vortex patch domains on the sphere exist. The aim here is to construct the spherical analogues of the planar multipolar vortices found in Crowdy. ${ }^{12} \mathrm{~A}$ typical $N+1$-polar solution in Ref. 12 consists of a patch of uniform vorticity with a central point vortex (at the patch centroid) and $N$ satellite point vortices symmetrically disposed about this central point vortex.

To construct the spherical analogues explicitly it is expedient to introduce a conformal mapping $\zeta(\eta)$ from a parametric $\eta$-plane mapping the unit $\eta$-disc to the (projected) vortex patch $D_{p}$ in the $\zeta$-plane. See Fig. 1 for a schematic. Let

$$
\zeta(\eta)=R\left(\eta+\frac{b \eta}{\eta^{N}-a^{N}}\right)
$$


where $R, a$ and $b$ are real constants with $a>1$ and $N \geqslant 2$ is an integer. It will be shown that, for suitable choices of the parameters $R, a, b$, and $N$, the maps (27) provide the appropriate conformal parametrization of the boundaries of a class of $(N+1)$-polar vortices in equilibrium on the surface of a sphere.

The condition $a>1$ is specified in order that the conformal map is analytic in the unit $\eta$-disc. Owing to the bijective nature of the stereographic projection, the requirement that the vortex patch boundary (on the sphere) should not cross itself translates to a condition that the conformal map (27) be a univalent conformal map from the unit $\eta$-circle. It is also assumed that the projected boundary of the vortex patch is an analytic curve. Note that

$$
\bar{\zeta}(\eta)=\zeta(\eta)
$$

where the conjugate function $\bar{\zeta}(\eta)$ is defined as

$$
\bar{\zeta}(\eta)=\overline{\zeta(\bar{\eta})} .
$$

In terms of the conformal mapping variable $\eta$, the Schwarz function can be written

$$
S(\zeta)=\zeta\left(\eta^{-1}\right)
$$

where we have used (28) and the fact that $\bar{\eta}=\eta^{-1}$ on $\partial D_{p}$. In terms of the variables $\eta, \bar{\eta}$, the function $\psi_{\zeta}$ takes the form

$$
\psi_{\zeta}=\left\{\begin{array}{c}
-\omega_{0}\left(\frac{\bar{\zeta}(\bar{\eta})}{(1+\zeta(\eta) \bar{\zeta}(\bar{\eta}))}\right. \\
\left.-\frac{\zeta\left(\eta^{-1}\right)}{\left(1+\zeta(\eta) \zeta\left(\eta^{-1}\right)\right)}\right), \quad|\eta| \leqslant 1 \\
0 \quad|\eta|>1
\end{array} .\right.
$$

By inspection, $S(\zeta(\eta))$ is seen to be analytic everywhere inside the unit $\eta$-circle except at $\eta=0$ and at all points $\eta$ (with $|\eta|<1$ ) satisfying

$$
1+\zeta(\eta) \zeta\left(\eta^{-1}\right)=0
$$

(32) is equivalent to the following quadratic equation for $\eta^{N}$ :

$$
c_{2}(a, b, R, N) \eta^{2 N}+c_{1}(a, b, R, N) \eta^{N}+c_{0}(a, b, R, N)=0,
$$

where

$$
\begin{aligned}
& c_{2}(a, b, R, N) \equiv R^{2}\left(b-a^{N}\right)-a^{N}, \\
& c_{1}(a, b, R, N) \equiv 1+a^{2 N}+R^{2}+R^{2}\left(b-a^{N}\right)^{2}, \\
& c_{0}(a, b, R, N) \equiv R^{2}\left(b-a^{N}\right)-a^{N} .
\end{aligned}
$$

It is instructive to observe that as $R \rightarrow 0$ (which, for point vortices situated close to the south pole, forces the vortex patch to be localized near the south pole) the solutions of (33) tend to $\eta^{N}=a^{-N}, a^{N}$. The solution $\eta^{N}=a^{N}$ is discarded because it yields $N$ solutions for $\eta$ which are not inside the unit $\eta$-circle, while the solution $\eta^{N}=a^{-N}$ retrieves the expected result for the planar case. ${ }^{12}$

This solution structure for (32) is found to be generic: Let $\eta_{s}$ denote the positive real root of (33) satisfying $\left|\eta_{s}\right|$ $<1$. There exist $N-1$ other symmetrically disposed zeros inside the unit circle with the same modulus and with argu- ments corresponding to the other $N$ th roots of unity. At all such zeros $\mathcal{S}(\zeta(\eta))$ has a simple pole singularity with a real residue. Together, these points correspond to the $\eta$-preimages of the $N$ satellite point vortices.

Let $\zeta_{s}$ denote the image of $\eta_{s}$ under the conformal map; i.e.,

$$
\zeta_{s}=\zeta\left(\eta_{s}\right)
$$

$\zeta_{s}$, therefore, corresponds to the stereographic projection of a (satellite) point vortex on the surface of the sphere. The singularity of $\mathcal{S}(\zeta(\eta))$ at $\eta=0$ corresponds to a point vortex at the south pole.

\section{B. Stationarity of the point vortices}

By construction, both the kinematic and dynamic boundary conditions on the vortex patch boundary have been satisfied. By the Helmholtz laws of vortex motion, it remains to ensure that all point vortices are stationary under the effects of the nonself-induced terms in the local velocity field. To impose this condition, it is most convenient to consider the local expansion of the velocity field in Cartesian components in the stereographically projected $\zeta$-plane. Near a satellite point vortex at $\zeta_{s}$, we can write

$$
\frac{\zeta\left(\eta^{-1}\right)}{1+\zeta(\eta) \zeta\left(\eta^{-1}\right)}=\frac{\Gamma_{s}}{\zeta-\zeta_{s}}+\gamma_{s}+\cdots
$$

for some real coefficients $\Gamma_{s}$ and $\gamma_{s}$ so that, near $\zeta_{s}$

$$
u-i v=-\frac{2 \omega_{0} \zeta}{\sin \theta}\left(\frac{\bar{\zeta}}{(1+\zeta \bar{\zeta})}-\frac{\Gamma_{s}}{\zeta-\zeta_{s}}-\gamma_{s}+\cdots\right) .
$$

To find expressions for $\Gamma_{s}$ and $\gamma_{s}$, define the auxiliary function

$$
G(\eta) \equiv 1+\zeta(\eta) \zeta\left(\eta^{-1}\right) .
$$

After some Taylor expansions and algebraic manipulations, it follows that

$$
\begin{aligned}
\Gamma_{s} & =\frac{\zeta\left(\eta_{s}^{-1}\right) \zeta_{\eta}\left(\eta_{s}\right)}{G_{\eta}\left(\eta_{s}\right)}, \\
\gamma_{s} & =\frac{\zeta\left(\eta_{s}^{-1}\right)}{G_{\eta}\left(\eta_{s}\right)}\left(\frac{\zeta_{\eta \eta}\left(\eta_{s}\right)}{2 \zeta_{\eta}\left(\eta_{s}\right)}-\frac{G_{\eta \eta}\left(\eta_{s}\right)}{2 G_{\eta}\left(\eta_{s}\right)}-\frac{\zeta_{\eta}\left(\eta_{s}^{-1}\right)}{\eta_{s}^{2} \zeta\left(\eta_{s}^{-1}\right)}\right) .
\end{aligned}
$$

In order the determine the condition for stationarity of the point vortex at $\zeta_{s}$, it is necessary to subtract off its selfinduced contribution to the velocity field. In terms of the stereographically projected coordinate, the streamfunction $\psi_{p}$ for a point vortex of strength $\kappa$ at $\zeta_{s}$ is

$$
\psi_{p}=-\frac{\kappa}{2} \log \left(\frac{\left(\zeta-\zeta_{s}\right)\left(\bar{\zeta}-\bar{\zeta}_{s}\right)}{(1+\zeta \bar{\zeta})\left(1+\zeta_{s} \bar{\zeta}_{s}\right)}\right) .
$$

See Appendix A for more details. Analogous to (16), the associated velocity field on the sphere is given as

$$
u_{p}-i v_{p}=\frac{2 \zeta}{\sin \theta} \psi_{p \zeta}=-\frac{2 \zeta}{\sin \theta}\left(\frac{\kappa}{2} \frac{1}{\zeta-\zeta_{s}}-\frac{\kappa}{2} \frac{\bar{\zeta}}{(1+\zeta \bar{\zeta})}\right),
$$


where $u_{p}$ and $v_{p}$, respectively, denote the zonal and meridional components of the velocity field induced by the point vortex. The first term on the right-hand side of (41) is singular at $\zeta=\zeta_{s}$ and corresponds to the point vortex singularity. The second term corresponds to the background of uniform vorticity which must exist in order to satisfy the global constraint (9). To see this, note that a uniform patch of vorticity of strength $\kappa / 2$ satisfies

$$
\nabla_{\Sigma}^{2} \psi=-\frac{\kappa}{2} .
$$

Using (15) this is equivalent to

$$
\psi_{\zeta \bar{\zeta}}=-\frac{\kappa}{2} \frac{1}{(1+\zeta \bar{\zeta})^{2}},
$$

which can be integrated with respect to $\bar{\zeta}$ to give

$$
\psi_{\zeta}=\frac{\kappa}{2}\left(\frac{1}{\zeta(1+\zeta \bar{\zeta})}+f(\zeta)\right),
$$

where $f(\zeta)$ is an arbitrary function. Picking $f(\zeta)=-1 / \zeta$ so that the velocity field is regular at $\zeta=0$, yields

$$
\psi_{\zeta}=\frac{\kappa}{2} \frac{\bar{\zeta}}{(1+\zeta \bar{\zeta})},
$$

which, using (16), gives precisely the second term on the right-hand side of (41). Comparing (37) with (41) reveals that the point vortex at $\zeta_{s}$ has strength

$$
\kappa_{s}=-2 \omega_{0} \Gamma_{s},
$$

while the condition for stationarity is

$$
\left.\left[(u-i v)-\left(u_{p}-i v_{p}\right)\right]\right|_{\left(\zeta_{s}, \bar{\zeta}_{s}\right)}=0,
$$

which is equivalent to

$$
\frac{\left(1-\Gamma_{s}\right) \bar{\zeta}_{s}}{\left(1+\zeta_{s} \bar{\zeta}_{s}\right)}-\gamma_{s}=0
$$

Equation (48) is henceforth referred to as the stationarity condition. It can be directly verified that, by the symmetry of the configuration and the associated velocity field, (48) is sufficient to ensure that all other symmetrically disposed point vortices are also stationary.

It can also be shown that, near $\zeta=0$

$$
\frac{\zeta\left(\eta^{-1}\right)}{1+\zeta(\eta) \zeta\left(\eta^{-1}\right)}=\frac{\Gamma_{c}}{\zeta}+\gamma_{c}+\cdots
$$

where

$$
\Gamma_{c}=\frac{R^{2}\left(1-b a^{-N}\right)}{1+R^{2}\left(1-b a^{-N}\right)},
$$

and $\gamma_{c}=0$. This means that there is a point vortex at the south pole and that it is automatically stationary so that no additional conditions need to be satisfied. The point vortex at the south pole has strength

$$
\kappa_{c}=-2 \omega_{0} \Gamma_{c} .
$$

It is worth remarking that, in the case of the exact solution class for multipolar vortices in the plane, ${ }^{12}$ the analogue to the stationarity condition (48) was independent of the parameter $R$ which can be thought of as a normalization parameter on the size of the vortex. This reflects the lack of a characteristic lengthscale in that problem and hence the fact that the length-scale of the vorticity distribution could be arbitrarily set. This is no longer true in the case of the sphere and its radius now defines a characteristic lengthscale. Consequently, the relevant stationarity condition (48) is now inextricably dependent on $R$. It is also noted that whereas the analogous nonlinear stationarity condition for the planar solutions could be solved exactly (leading to completely explicit solutions-see Ref. 13), this is no longer possible in the spherical case. Thus, although the solutions can be described in exactly in terms of a finite set of parameters, those parameters must be determined numerically.

\section{PROPERTIES OF THE SOLUTIONS}

To investigate solutions for a given $N$, it is natural to specify the latitudinal angle $\theta_{0}$ at which all the satellite vortices are situated. This corresponds to specifying the lengthscale of the vortex structure relative to the characteristic lengthscale of the sphere. The value of the parameter $a$ is then varied and solutions sought. For any given $a$ and $N$, the specified value of $\theta_{0}$ provides the value of $\zeta_{s}$ via the formula

$$
\zeta_{s}=\cot \left(\frac{\theta_{0}}{2}\right) .
$$

This provides one equation relating the three as yet undetermined parameters $R, b$ and $\eta_{s}$. A second relation derives from the condition of stationarity of the satellite vortices (48) while a third condition is Eq. (32). These three nonlinear equations are solved simultaneously for $R, b$ and $\eta_{s}$ using Newton's method.

Only $R$ and $b$ are required to actually reconstruct the vortex patch solutions. To plot the solutions on the physical sphere, the following relations are inverted for $\theta$ and $\phi$, viz:

$$
\begin{aligned}
& \cot \left(\frac{\theta}{2}\right)=|\zeta(\eta)|, \\
& \phi=\arg [\zeta(\eta)],
\end{aligned}
$$

for a discrete set of points on the $|\eta|=1$ circle. Use of the formulas

$$
\begin{aligned}
& x=\sin \theta \cos \phi, \\
& y=\sin \theta \sin \phi, \\
& z=\cos \theta,
\end{aligned}
$$

then produces the physical position of the vortex patch boundary on the spherical surface. Exactly the same procedure is used to find the satellite point vortex positions.

Exact solutions have been found to exist for any positive integer $N \geqslant 2$, the $N=2$ case corresponding to tripolar solutions and higher values of $N$ to higher-order multipolar structures. For purposes of illustrating the general features of the solution class, we present the quadrupolar case $N=3$ in de- 


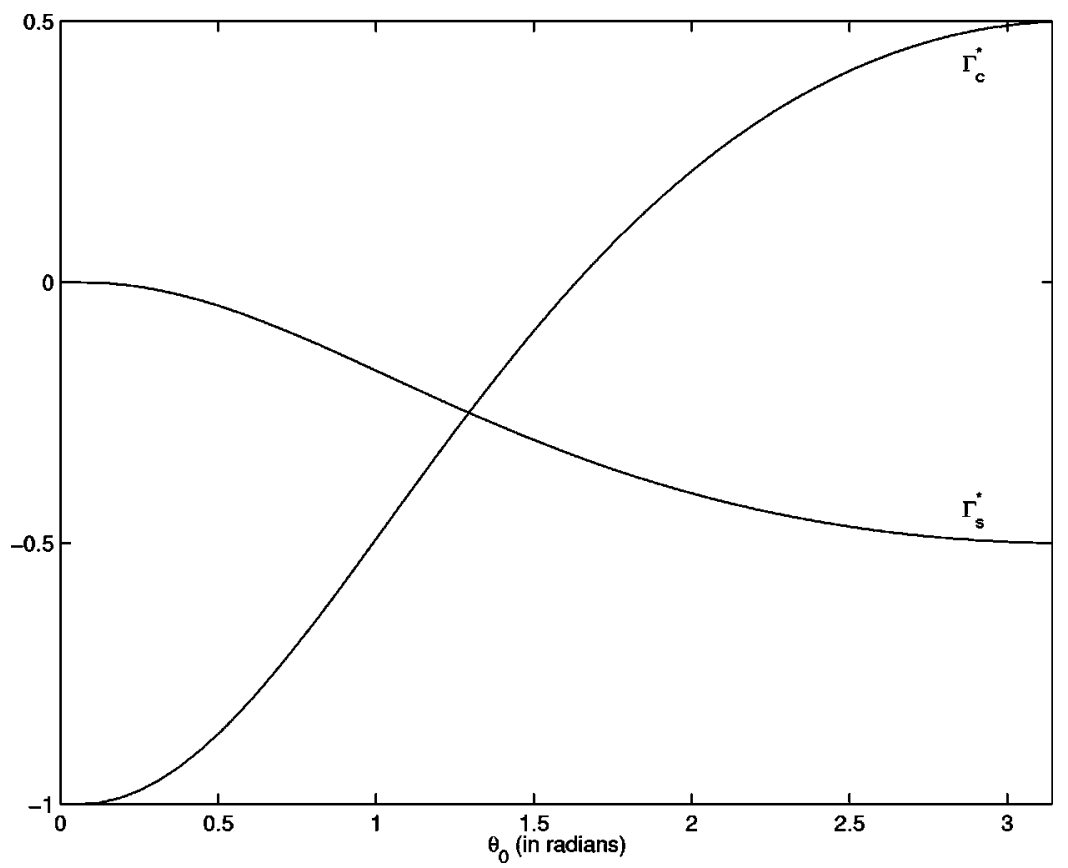

FIG. 2. $\Gamma_{c}^{*}$ and $\Gamma_{s}^{*}$ against latitudinal angle $\theta$ (in radians) for stationary point vortex problem with $N=3$. The graphs intersect at $\theta_{0}=\theta_{0}^{\text {crit }}=70.5^{\circ}$ (to 3 significant figures). tail. One reason for concentrating on the $N=3$ case is that a stability analysis ${ }^{13}$ of the planar configurations found in Crowdy $^{12}$ have revealed the quadrupolar solutions to be distinguished in that they are neutrally stable in all configurations except possibly those that are close to exhibiting cusps in the patch boundaries (the reader is referred to Crowdy and Cloke $^{13}$ for more details). The quadrupolar solutions are therefore the most likely solutions to be stable on the sphere (at least for certain configurations-see discussion section).

For fixed $N$ and $\theta_{0}$, it is found that varying $a$ corresponds to altering the area of the vortex patch. In all cases examined, the area of the vortex patch increases monotonically with $a$. Since the parameter $a$ has no obvious physical significance it is, therefore, more natural to plot solutions (for given $\theta_{0}$ ) as functions of the total vortex patch area $\mathcal{A}$. In Appendix $\mathrm{B}$ the formulas used to calculate $\mathcal{A}$ are given. It is also convenient to define $\Gamma_{p}$ as

$$
\Gamma_{p} \equiv \omega_{0} \mathcal{A}
$$

which represents the total strength of the vortex patch. All point vortex circulations are renormalized with respect to $\Gamma_{p}$. In the calculations which follow, we chose $\omega_{0}=1$.

It is found that for large $a$, the patch area tends to $4 \pi$ corresponding to a uniform blanketing of the whole sphere by the uniform patch vorticity. As $a$ decreases it can, therefore, be imagined that a growing region of quiescent irrotational fluid is developing at the north pole. This suggests a useful check on the problem formulation as well as the numerical method used to solve the nonlinear equations derived in the previous section. As $a$ gets large so that the entire sphere contains a uniform background patch of vorticity, the problem reduces to a point vortex problem on the sphere. This point vortex problem is the spherical generalization of the planar point vortex problem of Morikawa and Swenson ${ }^{18}$ where a central line vortex is placed at the south pole and $N$ equal-strength satellites are equispaced in longitudinal angle around some specified latitudinal circle at angle $\theta=\theta_{0}$. The strengths $\Gamma_{c}^{*}$ and $\Gamma_{s}^{*}$, say, of the central and satellite vortices, respectively, must be chosen in such a way that the satellites are stationary (for general values of the central line vortex circulation one would obtain a relative equilibrium in which the satellites rotate at constant angular velocity). The present authors have not found the solution to this point vortex problem written down in the literature. It is therefore solved in Appendix C, using stereographically projected coordinates, and the values of $\Gamma_{s}^{*}$ and $\Gamma_{c}^{*}$ are found explicitly as functions of $N$ and $\theta_{0}$. A graph of $\Gamma_{s}^{*}$ and $\Gamma_{c}^{*}$ against $\theta_{0}$ is shown in Fig. 2.

Figure 2 is instructive because it indicates that, in the point vortex problem, the point vortex at the south pole is of the same (positive) strength as the uniform background vorticity when the satellites are close to it in latitude. As the satellites move further away from the south pole, the strength of the point vortex at the south pole goes through zero when the satellites are at the equator and becomes negative when the satellites are in the northern hemisphere. The generalized solutions found here are expected to behave similarly. Figures 3 and 4 show graphs of $\Gamma_{c} / \Gamma_{p}$ and $\Gamma_{s} / \Gamma_{p}$ against patch area $\mathcal{A}$ for various choices of $\theta_{0}$. These quantities determine the dynamical nature of the flow because they measure the point vortex strengths relative to the total background patch vorticity. For any given $\theta_{0}$, these graphs terminate at some critical area below which no solution can be found. The corresponding limiting solutions display cuspidal singularities in the patch boundary and are discussed in more detail shortly. As expected, at large latitude angles (so that the satellites are in the southern hemisphere and close to the southern polar point vortex), the graphs in Figs. 3 and 4 are qualitatively similar to those plotted for the planar case in Ref. 13. However, as the latitudinal angle of the satellites decreases (so that the satellites move towards the northern hemisphere) 


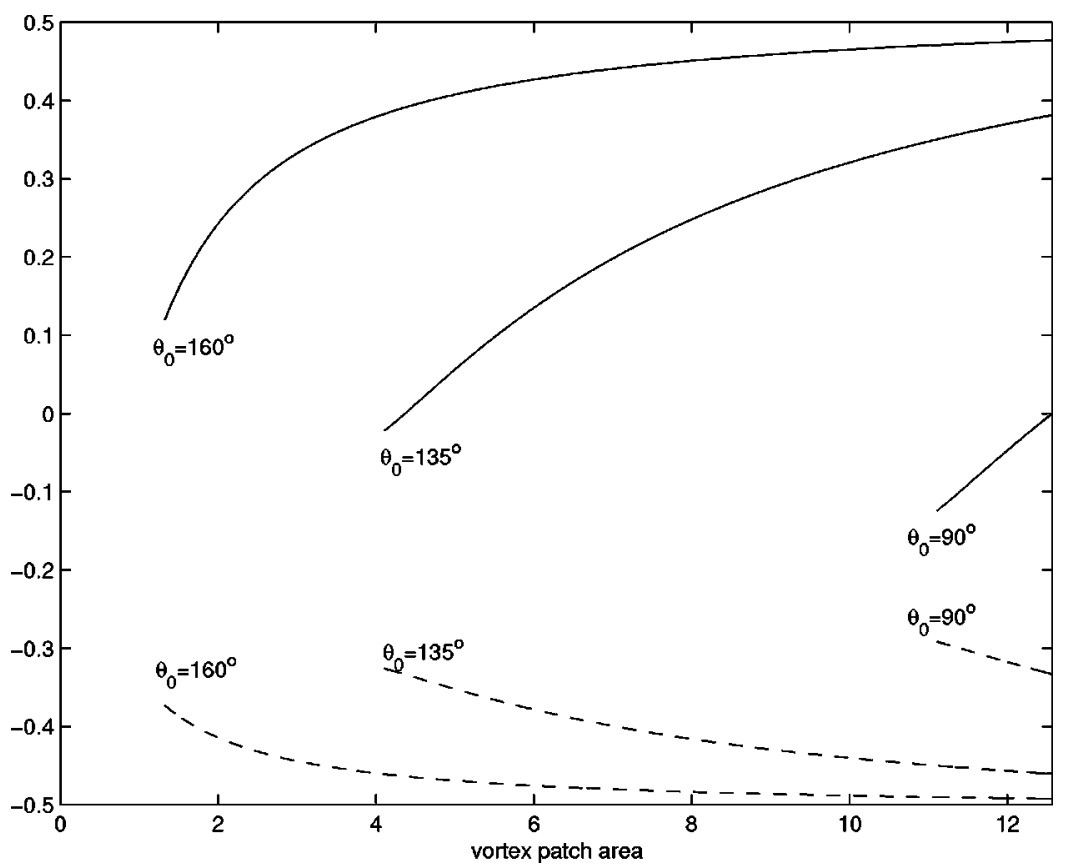

FIG. 3. Renormalized point vortex strengths $\Gamma_{c} / \Gamma_{p}$ (solid lines) and $\Gamma_{s} / \Gamma_{p}$ (dashed lines) against vortex patch area for $N=3$ and $\theta_{0}=160^{\circ}, 135^{\circ}$, and $90^{\circ}$. The values at patch area equal to $4 \pi$ correspond to those given in Fig. 2. a "cross-over" occurs as the relative strength of the point vortex at the south pole changes from positive to negative. At $\theta_{0}=\theta_{0}^{\text {crit }}=70.5^{\circ}$ (a value obtained by equating $\Gamma_{c}^{*}=\Gamma_{s}^{*}$ using the expressions (C4) and (C5) obtained in Appendix C) the value of $\Gamma_{c} / \Gamma_{p}$ equals $\Gamma_{s} / \Gamma_{p}$ when the patch has maximum area so that all point vortices have the same strength. $\theta_{0}^{\text {crit }}$ represents a cross-over latitude where the point vortex at the south pole is becoming just as important as the satellites in cancelling out the overall uniform background vorticity.

For each $N$ and $\theta_{0}$, a critical value of the patch area exists for which physically admissible equilibria can be found. This critical value provides a lower bound on the admissible values of the patch area. The corresponding limiting states exhibit cusps in the vortex patch boundary. An analogous situation arises in the planar case. ${ }^{13}$ The neighborhood of any point on a sphere is locally planar so this observation on the limiting states is consistent with a local analysis of Overman ${ }^{19}$ who studied limiting V-states in the plane and found that any points of nonanalyticity in the patch boundary must be either $90^{\circ}$-corners or cusps.

In Fig. 5 the solutions are shown in orthographic projection on the physical sphere in the case $\theta_{0}=160^{\circ}$ and for four different choices of patch area. The associated stereographic projections are also shown to the right of each orthographic projection. The smallest patch area $\mathcal{A}=0.868$ (correct to 3 decimal places) is close to the limiting cuspidal configuration, while the largest area shown $(\mathcal{A}=11.805)$ is close to the pure point vortex case where the patch entirely covers the

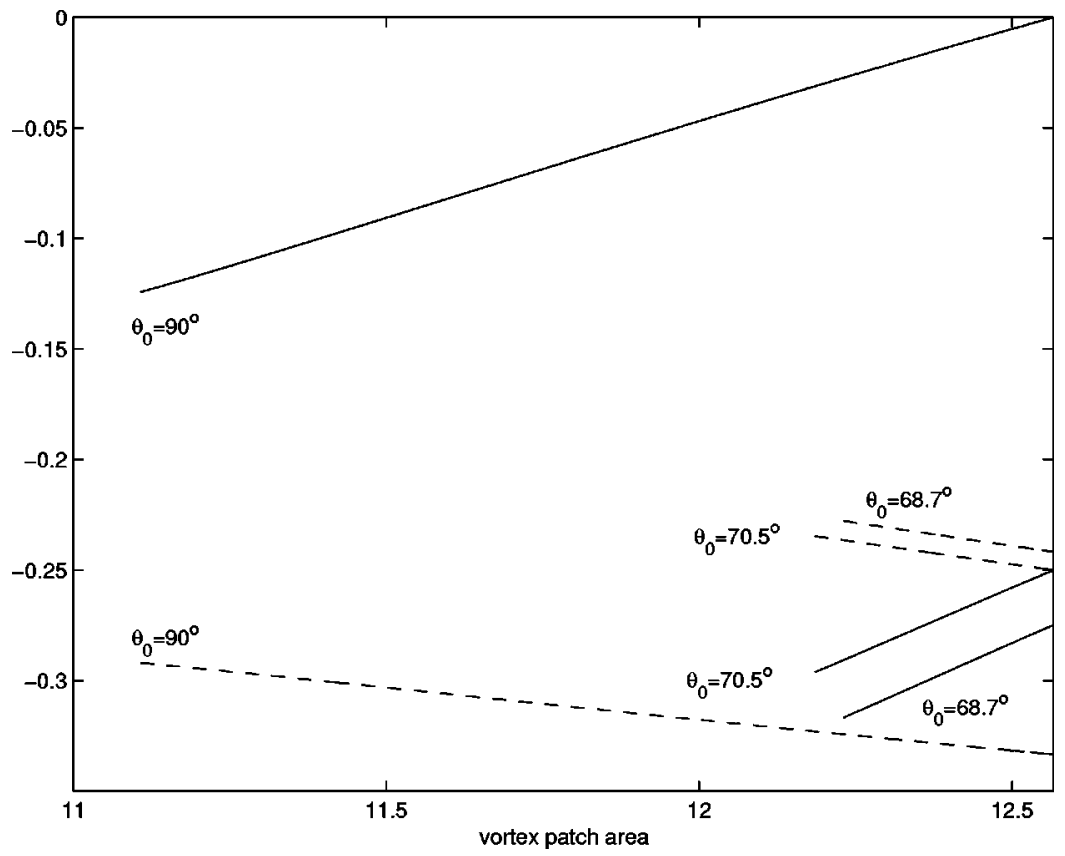

FIG. 4. Renormalized point vortex strengths $\Gamma_{c} / \Gamma_{p}$ (solid lines) and $\Gamma_{s} / \Gamma_{p}$ (dashed lines) against vortex patch area for $N=3$ and $\theta_{0}=90^{\circ}, 70.5^{\circ}\left(=\theta_{0}^{\text {crit }}\right)$ and $68.7^{\circ}$. The values at patch area equal to $4 \pi$ correspond to those given in Fig. 2. 

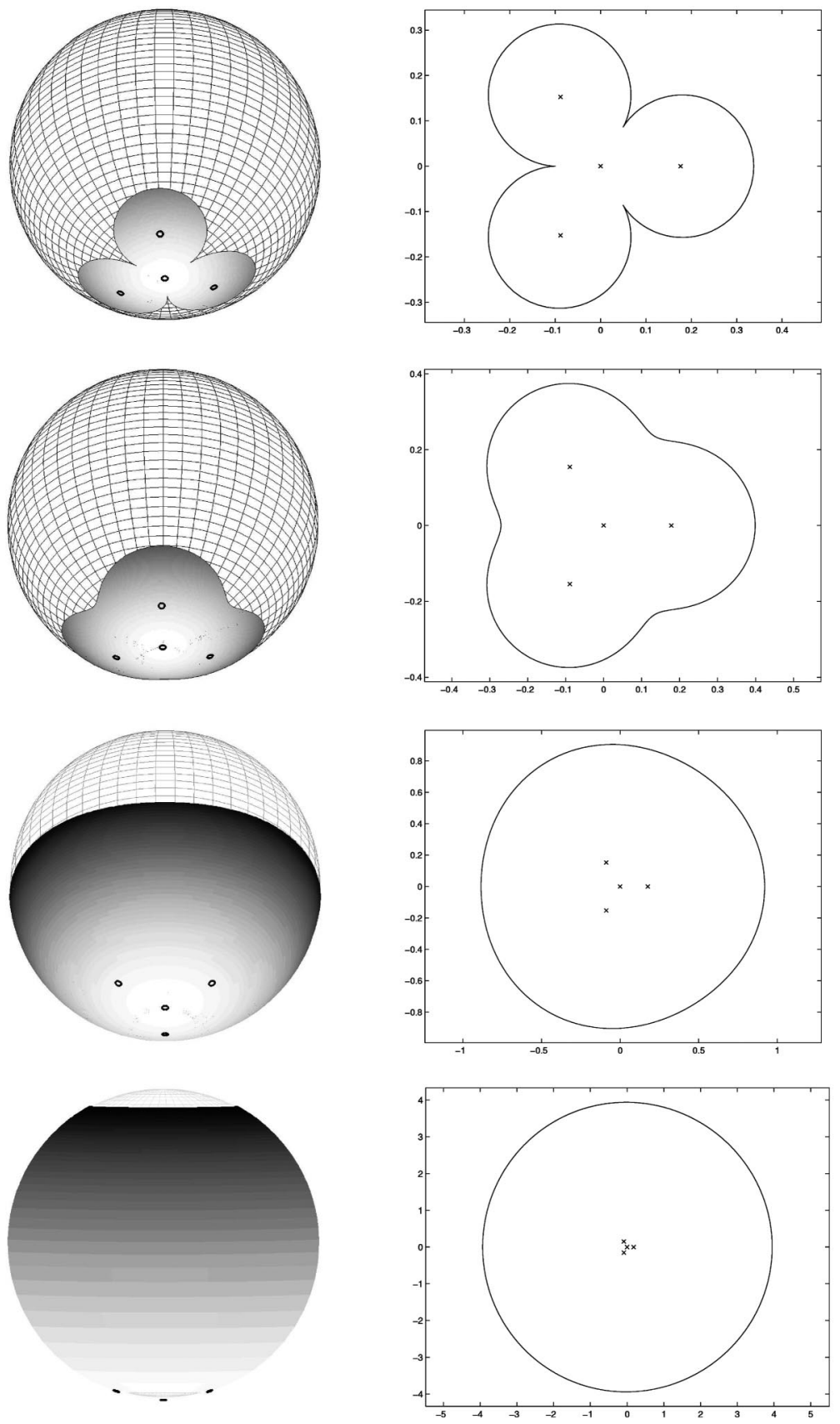

FIG. 5. Multipolar vortices with $N=3$ satellites at latitude $\theta_{0}=160^{\circ}$ for different vortex patch areas $\mathcal{A}$ $=0.868$ (close to cusped configuration), 1.322, 5.640, and 11.805 (close to point-vortex case). Each solution is shown in orthographic projection on the left and in stereographic projection on the right. The corresponding point vortex strengths $\left(\Gamma_{c}, \Gamma_{s}\right)$ are given, respectively, by $(0.001,0.022),(-0.013,0.039),(-0.189,0.213)$, and $(-0.445,0.462)$.

sphere. Two intermediate shapes with different areas are also shown. The orthographic projection in these diagrams has been varied in order to highlight the structural features of each solution. Figure 6 shows three typical vortex equilibria for the case $N=3$ and $\theta_{0}=135^{\circ}$ while Fig. 7 shows typical equilibria for $N=3$ and $\theta_{0}=90^{\circ}$ so that the satellites are on the equator. As $\theta_{0}$ decreases, the range of possible patch areas for which solutions can be found decreases as is evident from Figs. 3 and 4.

Finally, for illustration, some examples of tripolar $N$ $=2$ solutions are shown in Fig. 8 for point vortices at $\theta_{0}$ $=160^{\circ}$ while Fig. 9 shows similar results for the pentapolar case $N=4$.

\section{DISCUSSION}

A broad class of analytical solutions for distributed regions of vorticity in equilibrium on a sphere has been presented. The construction is a generalization of the mathematical approach originally expounded in Crowdy ${ }^{12}$ to study planar multipolar equilibria. This generalization is significant given that other well-known planar results (e.g., the exact solution for the rotating Kirchhoff ellipse) do not generalize to the spherical geometry.

The solutions are presented as a contribution to the mathematical theory of vortex dynamics on a sphere. The theoretical approach is potentially generalizable to the con- 

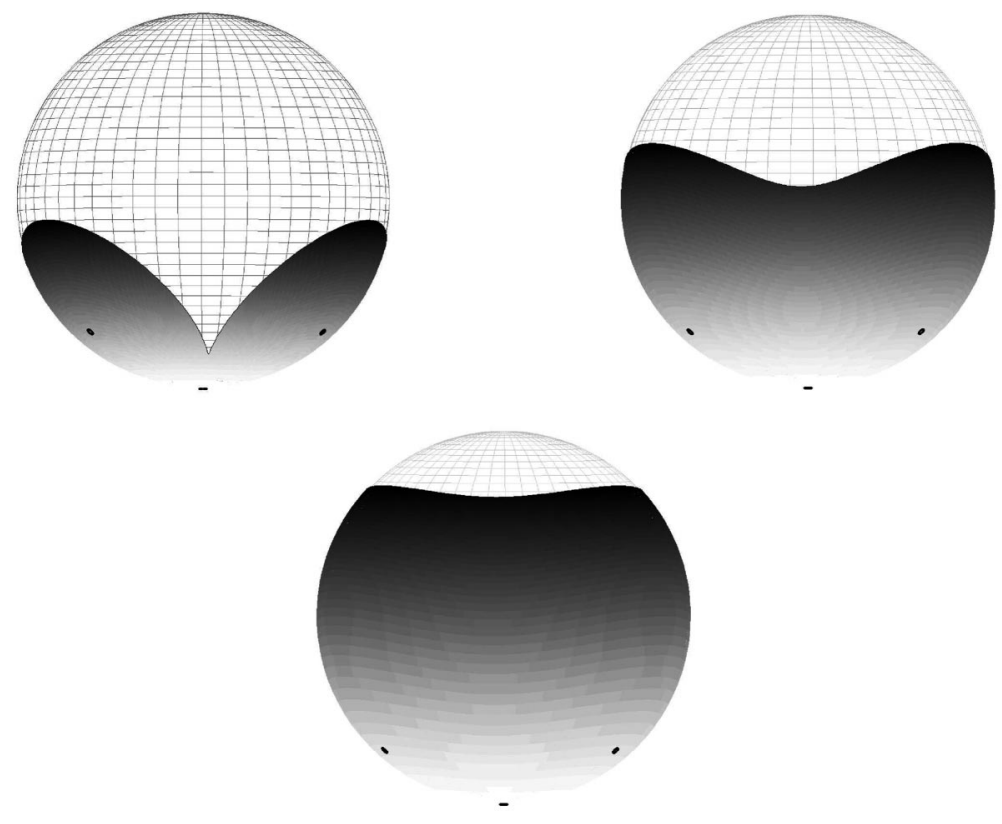

struction of distributed vortex equilibria on more general closed surfaces. Other generalizations might be feasible; Crowdy ${ }^{20}$ has extended the approach of Ref. 12 to construct rotating vortex equilibria in the plane which generalize the classical results of Thomson ${ }^{21}$ and Morikawa and Swenson. ${ }^{18}$ Thus, it may similarly be possible to generalize such planar rotating equilibria to the sphere.

The stability of the spherical multipolar equilibria is of interest, especially the question of how these stability properties compare to those of the planar case (which has recently been examined in detail by Crowdy and Cloke ${ }^{13}$ ). Although the linear stability calculation is made easier by knowledge of closed-form formulas for the equilibrium base-states, it is nevertheless somewhat involved and beyond the scope of the present paper. It can be anticipated that at least some of the equilibria found here will be linearly stable. For example, the planar quadrupolar solutions of Crowdy ${ }^{12}$ have recently been found to be neutrally stable in all configurations ${ }^{13}$ (except possibly those near to limiting where the question of linear stability could not be resolved using the method of Ref. 13). For small-area patches with satellites close to the south pole, the spherical quadrupolar solutions are close to the planar case (in the sense that the spherical curvature will have little effect on such solutions). They are therefore similarly expected to be stable. How these linear stability properties change as the quadrupoles grow bigger and cover more of the spherical surface, as well as how they depend on the latitudinal positions of the satellite point vortices, are interesting questions. Dritschel and Polvani ${ }^{4}$ have found that distributions of vorticity on the sphere can have very different stability properties to their planar analogues.

Since the new solutions consist of combinations of uniform vortex patches with superposed point vortices, the nonlinear stability and nonlinear evolution of these configurations can be studied by simple adaptations of existing contour dynamics/surgery codes on a sphere. ${ }^{22}$ It is simply necessary to add a finite set of ordinary differential equations governing the point vortex motion to these codes. At the same time, the analytical solutions herein provide important nontrivial checks on such numerical codes. This feature also provides a possible alternative to performing the detailed linear stability calculation mentioned above; Polvani and Dritschel, ${ }^{4}$ for example, have studied the stability of a class of (numerically computed) vortex patch equilibria on the sphere using contour dynamics to observe the behavior of slightly perturbed equilibria and the same method can, in principle, be used in respect of the present solutions.

On a mathematical note, the present authors have not seen the simple expression (A7) for the streamfunction $\psi$ associated with a point vortex of strength $\kappa$ (derived in Ap-
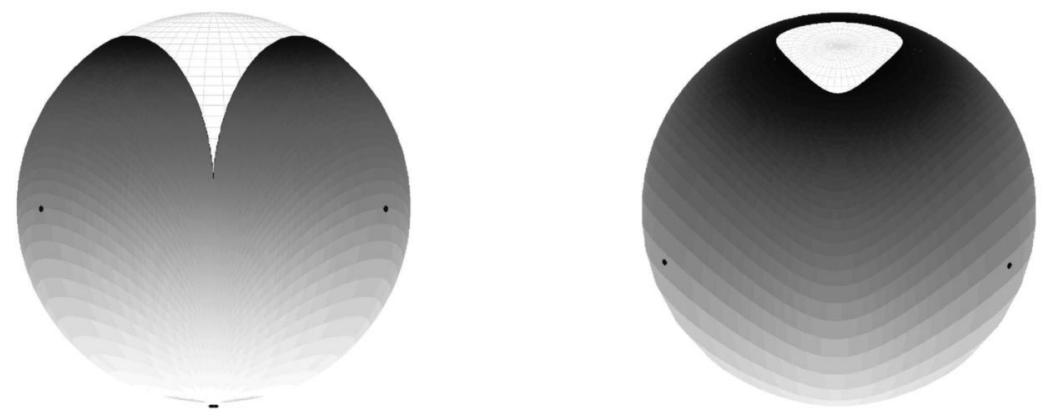

FIG. 7. Multipolar vortices with $N=3$ satellites at latitude $\theta_{0}=90^{\circ}$ for different vortex patch areas $\mathcal{A}$ $=11.107$ and 12.271. The corresponding point vortex strengths $\left(\Gamma_{c}, \Gamma_{s}\right)$ are given, respectively, by $(0.110$, $0.258)$ and $(0.024,0.318)$. 

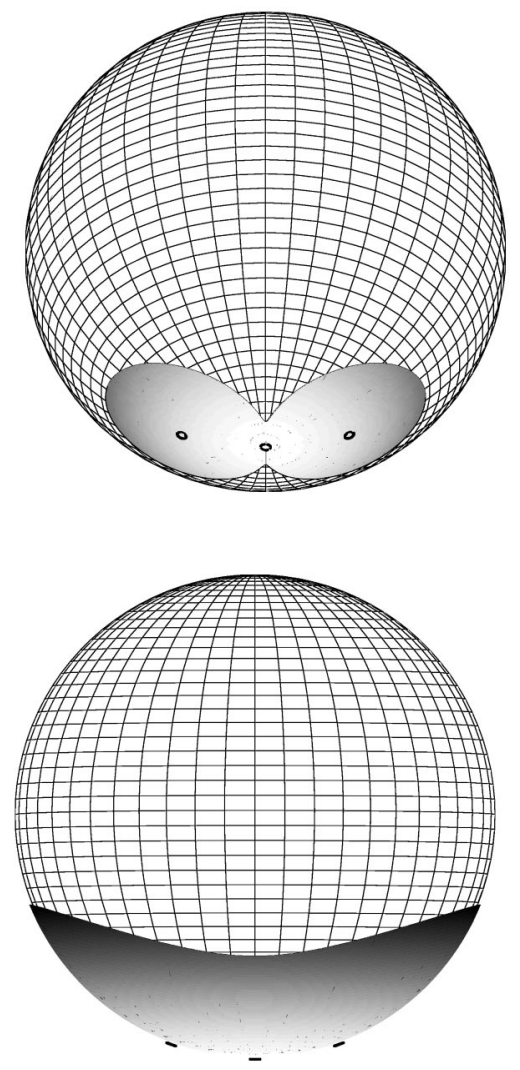

FIG. 8. Multipolar vortices with $N=2$ satellites at latitude $\theta_{0}=160^{\circ}$ for vortex patch areas $\mathcal{A}=0.846,3.272$, and 11.007. The corresponding point vortex strengths $\left(\Gamma_{c}, \Gamma_{s}\right)$ are given, respectively, by $(-0.001,0.034)$, $(-0.056,0.159)$, and $(-0.275,0.575)$.

pendix A) explicitly written down in the literature in terms of the complex stereographic coordinate $\zeta$. Indeed, the use of stereographic projection to study the dynamics of vorticity on a sphere is rare although Dritschel ${ }^{23}$ and Kimura ${ }^{24}$ employ it in various contexts. Bogomolov ${ }^{2}$ also mentions the possibility of studying spherical point vortex dynamics in this way. Stereographic projection, combined with conformal mapping theory, has been crucial in the construction of the present solutions and provides a particularly convenient parametrization thereof. The new solutions would have an extremely complicated mathematical representation if rewritten in terms of the original spherical polar angle variables $\phi$ and $\theta$.

\section{APPENDIX A: FUNDAMENTAL SOLUTIONS AND POINT VORTICES}

Let the stereographic projections of two points on the sphere with spherical polar coordinates $(1, \theta, \phi)$ and $\left(1, \theta^{\prime}, \phi^{\prime}\right)$ be $\zeta$ and $\zeta^{\prime}$, respectively. The simplest fundamental solution (see, e.g., Ref. 3) of

$$
\nabla_{\Sigma}^{2} \psi=\delta\left(\theta, \phi, \theta^{\prime}, \phi^{\prime}\right)-\frac{1}{4 \pi},
$$

satisfying the constraint

$$
\int \omega d \sigma=-\int \nabla_{\Sigma}^{2} \psi d \sigma=0,
$$
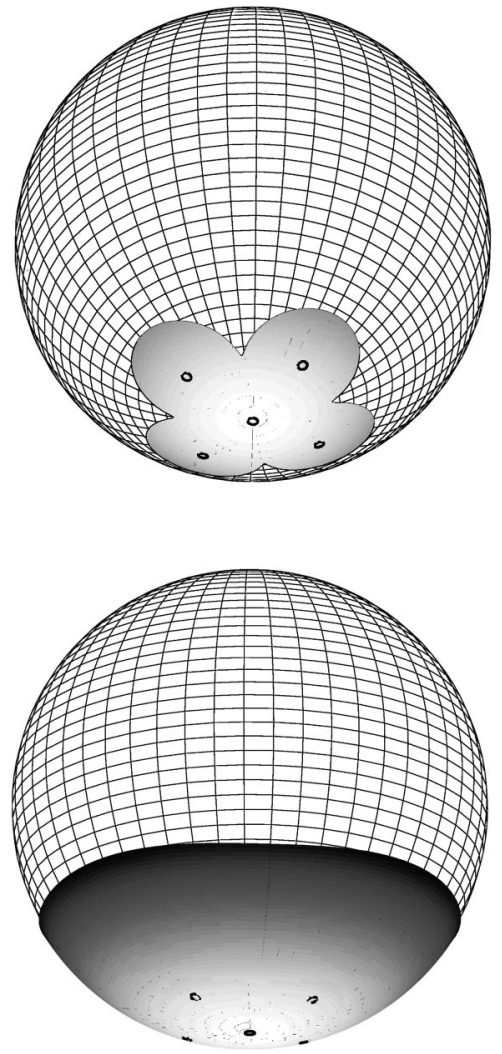

FIG. 9. Multipolar vortices with $N=4$ satellites at latitude $\theta_{0}=160^{\circ}$ for vortex patch areas $\mathcal{A}=0.838$ and 3.239. The corresponding point vortex strengths $\left(\Gamma_{c}, \Gamma_{s}\right)$ are given, respectively, by $(0.003,0.016)$ and $(-0.111$, $0.092)$.

where $d \sigma$ is the area element on the surface of the sphere and integration is over the entire spherical surface, is given by

$$
\psi=\frac{1}{4 \pi} \log (1-\cos \gamma),
$$

where

$$
\cos \gamma \equiv \cos \theta \cos \theta^{\prime}+\sin \theta \sin \theta^{\prime} \cos \left(\phi-\phi^{\prime}\right) .
$$

After some algebra, it can be shown that

$$
1-\cos \gamma=\frac{2\left(\zeta-\zeta^{\prime}\right)\left(\bar{\zeta}-\bar{\zeta}^{\prime}\right)}{(1+\zeta \bar{\zeta})\left(1+\zeta^{\prime} \bar{\zeta}^{\prime}\right)} .
$$

Therefore, the streamfunction associated with a point vortex of unit circulation at $\zeta_{s}$ is given, as a function of $\zeta$ and $\bar{\zeta}$, by

$$
\psi=-\frac{1}{4 \pi} \log \left(\frac{2\left(\zeta-\zeta_{s}\right)\left(\bar{\zeta}-\bar{\zeta}_{s}\right)}{(1+\zeta \bar{\zeta})\left(1+\zeta_{s} \bar{\zeta}_{s}\right)}\right)
$$

Alternatively, it is said that the streamfunction associated with a point vortex of strength $\kappa$ is

$$
\psi=-\frac{\kappa}{2} \log \left(\frac{2\left(\zeta-\zeta_{s}\right)\left(\bar{\zeta}-\bar{\zeta}_{s}\right)}{(1+\zeta \bar{\zeta})\left(1+\zeta_{s} \bar{\zeta}_{s}\right)}\right),
$$

where the circulation $\Gamma$ is related to the strength $\kappa$ via 


$$
\kappa=\frac{\Gamma}{2 \pi} .
$$

\section{APPENDIX B: COMPUTATION OF PATCH AREA}

The area element $d \sigma$ in spherical polars on a unit-radius sphere is

$$
d \sigma=\sin \theta d \theta d \phi .
$$

If $x$ and $y$ denote the Cartesian coordinates of $\zeta$ (so that $\zeta$ $=x+i y)$ then

$$
d x d y=|J(x, y ; \theta, \phi)| d \theta d \phi,
$$

where the Jacobian $J(x, y ; \theta, \phi)$ is

$$
\begin{aligned}
J(x, y ; \theta, \phi) & \equiv \frac{\partial(x, y)}{\partial(\theta, \phi)} \\
& =\frac{\sin \theta}{(1-\cos \theta)^{2}} .
\end{aligned}
$$

Using (12), the area $\mathcal{A}$ is therefore given by

$$
\begin{aligned}
\mathcal{A} & =\iint_{\tilde{D}} d \sigma \\
& =\iint_{D} \frac{4 d x d y}{(1+\zeta \bar{\zeta})^{2}} \\
& =\iint_{D} \frac{\partial}{\partial \bar{\zeta}}\left(\frac{4 \bar{\zeta}}{(1+\zeta \bar{\zeta})}\right) d x d y \\
& =\frac{1}{2 i} \oint_{\partial D} \frac{4 \bar{\zeta}}{(1+\zeta \bar{\zeta})} d \zeta,
\end{aligned}
$$

where the last line follows by application of the complex form of Green's theorem. More conveniently, in terms of the conformal mapping variable, the patch area is given by

$$
\mathcal{A}=\frac{1}{2 i} \oint_{|\eta|=1} \frac{4 \zeta\left(\eta^{-1}\right) \zeta_{\eta}(\eta)}{1+\zeta(\eta) \zeta\left(\eta^{-1}\right)} d \eta
$$

As a check, note that the special case $\zeta(\eta)=\eta$ corresponds to a hemispherical shell on a unit-radius sphere which is well-known to have area $2 \pi$.

A more convenient way to compute the area is to observe that

$$
\omega_{0} \mathcal{A}=4 \pi\left(N \Gamma_{s}+\Gamma_{c}\right),
$$

where $\Gamma_{s}$ and $\Gamma_{c}$ are defined in (39) and (50), respectively.

\section{APPENDIX C: STATIONARY POINT VORTEX CONFIGURATIONS}

Consider a point vortex configuration on sphere consisting of $N$ satellite point vortices of circulation $\Gamma_{s}^{*}$ equispaced in azimuthal angle $\phi$ around a fixed latitude circle $\theta^{\prime}=\theta_{0}$ with a circulation $\Gamma_{c}^{*}$ point vortex fixed at the south pole. Suppose that, at some instant, the satellite point vortices are at (projected) positions $\zeta_{j}=\zeta_{s} \omega_{N}^{j}$ where $\zeta_{s}=\cot \left(\theta_{0} / 2\right)$ and
$\omega_{N}=e^{2 \pi i / N}$. Then the instantaneous streamfunction is given, as a function of the stereographically projected coordinates, by

$$
\begin{aligned}
\psi(\zeta, \bar{\zeta})= & -\frac{\Gamma_{c}^{*}}{4 \pi} \log \left(\frac{\zeta \bar{\zeta}}{1+\zeta \bar{\zeta}}\right) \\
& -\frac{\Gamma_{s}^{*}}{4 \pi} \sum_{j=0}^{N-1} \log \left(\frac{\left(\zeta-\zeta_{j}\right)\left(\bar{\zeta}-\bar{\zeta}_{j}\right)}{(1+\zeta \bar{\zeta})\left(1+\zeta_{j} \bar{\zeta}_{j}\right)}\right) .
\end{aligned}
$$

Assume, without loss of generality, that the integral of the uniform background vorticity is equal to unity (so that the vorticity is locally equal to $1 / 4 \pi$ everywhere). The global constraint on the vorticity distribution, therefore, dictates that

$$
\Gamma_{c}^{*}+N \Gamma_{s}^{*}+1=0 .
$$

We seek solutions which are completely stationary (i.e., nonrotating). By the rotational symmetry of the configuration, it is enough to consider the point vortex at $\zeta_{s}$ (corresponding to $j=0$ ). By the Helmholtz vortex theorems, the condition that the nonself-induced component of the local velocity field at this point vortex is zero is equivalent to the condition

$$
\left.\left[\frac{\Gamma_{c}^{*}}{\zeta}-\frac{\Gamma_{c}^{*} \bar{\zeta}}{1+\zeta \bar{\zeta}}+\sum_{j=1}^{N-1}\left[\frac{\Gamma_{s}^{*}}{\zeta-\zeta_{s} \omega_{N}^{j}}-\frac{\Gamma_{s}^{*} \bar{\zeta}}{1+\zeta \bar{\zeta}}\right]\right]\right|_{\zeta=\bar{\zeta}=\zeta_{s}}=0 .
$$

(C2) and (C3) provide two equations for $\Gamma_{s}^{*}$ and $\Gamma_{c}^{*}$ as functions of $N$ and $\zeta_{s}$. Solving these equations gives

$$
\Gamma_{s}^{*}=-\frac{2}{(N+1)+(N-1) \zeta_{s}^{2}}
$$

and

$$
\Gamma_{c}^{*}=-\frac{(N-1)\left(\zeta_{s}^{2}-1\right)}{(N+1)+(N-1) \zeta_{s}^{2}} .
$$

The values given in (C4) and (C5) are precisely the values to which the renormalized circulations $\Gamma_{s} / \Gamma_{p}$ and $\Gamma_{c} / \Gamma_{p}$ should tend (respectively) as the patch area tends to $4 \pi$.

A graph of $\Gamma_{s}^{*}$ and $\Gamma_{c}^{*}$ against latitudinal angle $\theta_{0}$ is shown in Fig. 2 for the case $N=3$. When $\theta_{0}=\pi$ (so that all point vortices are superposed at the south pole) all satellites have circulation $-1 / 2$ while the central line vortex has circulation $1 / 2$. The total net circulation at the south pole is, therefore, equal to -1 , as must be the case to cancel out the total patch vorticity. As $\theta_{0}$ decreases (so that the satellite move further up the sphere), the satellites grow weaker until they have zero strength when at the north pole while the central vortex strength increases until it becomes positive and eventually reaches unity as the satellites reach the north pole. If the satellites have zero strength there, this must be the case because the point vortex at the south pole is then the only one "cancelling out" the overall patch vorticity.

${ }^{1}$ R. Kidambi and P. K. Newton, "Motion of three point vortices on a sphere," Physica D 116, 143 (1998).

${ }^{2}$ V. A. Bogomolov, "Dynamics of vorticity on a sphere," Fluid Dyn. 6, 863 (1977). 
${ }^{3}$ Y. Kimura and H. Okamoto, "Vortex motion on a sphere," J. Phys. Soc. Jpn. 56, 4203 (1987).

${ }^{4}$ D. G. Dritschel and L. M. Polvani, "The roll-up of vorticity strips on the surface of a sphere," J. Fluid Mech. 234, 47 (1992).

${ }^{5}$ L. M. Polvani and D. G. Dritschel, "Wave and vortex dynamics on the surface of a sphere," J. Fluid Mech. 255, 35 (1993).

${ }^{6}$ M. T. DiBattista and L. M. Polvani, "Barotropic vortex pairs on a rotating sphere," J. Fluid Mech. 358, 107 (1998).

${ }^{7}$ W. T. M. Verkley, "The construction of barotropic modons on a sphere," J. Atmos. Sci. 41, 2492 (1984).

${ }^{8}$ R. D. Pingree and B. LeCann, "Three anticyclonic slope water oceanic eddies (swoddies) in the southern Bay of Biscay," Deep-Sea Res., Part A 39, 1147 (1992).

${ }^{9}$ G. J. F. Van Heijst and R. Kloosterziel, "Tripolar vortices in a rotating fluid," Nature (London) 338, 569 (1989).

${ }^{10}$ G. F. Carnevale and R. Kloosterziel, "Emergence and evolution of triangular vortices,” J. Fluid Mech. 259, 305 (1994).

${ }^{11}$ Y. G. Morel and X. J. Carton, "Multipolar vortices in two-dimensional incompressible flows," J. Fluid Mech. 267, 23 (1994).

${ }^{12}$ D. Crowdy, "A class of exact multipolar vortices," Phys. Fluids 11, 2556 (1999).

${ }^{13}$ D. G. Crowdy and M. Cloke, "Stability analysis of a class of exact multipolar vortex equilbria," Phys. Fluids 14, 1862 (2002).
${ }^{14}$ D. G. Crowdy, "The construction of exact multipolar equilibria of the two-dimensional Euler equations," Phys. Fluids 14, 257 (2002).

${ }^{15}$ D. G. Crowdy, "Multipolar vortices and algebraic curves," Proc. R. Soc. London, Ser. A 457, 2337 (2001).

${ }^{16}$ P. G. Saffman, Vortex Dynamics (Cambridge University Press, Cambridge, 1992).

${ }^{17}$ P. J. Davis, The Schwarz Function and its Applications, Carus Mathematical Monographs (The Mathematical Association of America, Washington, DC, 1974).

${ }^{18}$ G. K. Morikawa and E. V. Swenson, "Interacting motion of rectilinear geostrophic vortices," Phys. Fluids 14, 1058 (1971).

${ }^{19}$ E. A. Overman, "Steady-state solutions of the Euler equations in two dimensions; Local analysis of limiting V-states," SIAM (Soc. Ind. Appl. Math.) J. Appl. Math. 46, 765 (1986).

${ }^{20}$ D. G. Crowdy, "Exact solutions for rotating vortex arrays with finite-area cores," J. Fluid Mech. 469, 209 (2002).

${ }^{21}$ J. J. Thomson, A Treatise on the Motion of Vortex Rings (Macmillan, New York, 1883).

${ }^{22}$ D. G. Dritschel, “Contour dynamics/surgery on the sphere," J. Comput. Phys. 79, 477 (1988).

${ }^{23}$ D. G. Dritschel, "A fast contour dynamics method for many-vortex calculations in two-dimensional flows," Phys. Fluids A 5, 173 (1993).

${ }^{24}$ Y. Kimura, "Vortex motion on surfaces with constant curvature," Proc. R. Soc. London, Ser. A 455, 245 (1999). 